\title{
Actualización del conocimiento del género Cyttaria Berk. (Cyttariales, Ascomycota) en Chile
}

(Note on knowledge of the genus Cyttaria Berk. (Cyttariales, Ascomycota) in Chile)

Salazar-Vidal, $\mathrm{V}^{.1,2 *}$

${ }^{1}$ Laboratorio de Química de Productos Naturales, Facultad de Ciencias Naturales y Oceanográficas, Universidad de Concepción. Víctor Lamas N 1290, Concepción, Chile. ${ }^{2}$ ONG Micófilos. Avenida Las Torres 851, San Pedro de la Paz, Chile. *Autor para correspondencia: vivianasalazar@udec.cl

RECIBIDO: 28 de marzo 2020 APROBADO: 22 de mayo 2020

DOI: $10.22370 /$ bolmicol.2020.35.1.2397

Palabras claves: Digüeñes, hongos comestibles, morfología, ecología, propiedades bioactivas.

Keywords: Digüeñes, edible fungi, morphology, ecology, bioactive properties.

\section{RESUMEN}

El género Cyttaria pertenece a la familia Cyttariaceae y sus especies son parásitos obligados de árboles del género Nothofagus. Se distribuye naturalmente en el hemisferio sur, encontrándose siete especies de Cyttaria presentes en nuestro país: $C$. berteroi, $C$. darwinii, C. espinosae, C. hariotii, C. hookeri, $C$. johowii y $C$. exigua, que comúnmente se conocen como "Digüeñes" y que tienen una gran importancia desde el punto de vista alimenticio, ya que han sido consumidos desde la prehistoria por pueblos originarios. En Chile se han realizado estudios sobre la taxonomía, ecología y propiedades medicinales de algunas especies del género Cyttaria y en esta revisión, se describen los caracteres macro y microscópicos e información relevante de las siete especies de este género presentes en nuestro país, de acuerdo a la literatura disponible y observaciones personales de la autora. Además, se presenta un resumen sobre los resultados de un estudio reciente de las propiedades bioactivas de las especies más consumidas en nuestro país.

\begin{abstract}
The genus Cyttaria belongs to the family Cyttariaceae; its species are obligate parasites of trees of the genus Nothofagus. It is naturally distributed in the Southern Hemisphere, with seven species of Cyttaria present in Chile: $C$. berteroi, C. darwinii, C. espinosae, C. hariotii, $C$. hookeri, $C$. johowii and $C$. exigua, which are commonly known as "Digüeñes" and are of great nutritional importance, since they have been consumed from prehistory by native people. Studies have been carried out in Chile on the taxonomy, ecology and medicinal properties of some species of the genus Cyttaria. In this review, the macro and microscopic characteristics and relevant information of the seven species of this genus present in our country are described, according to the available literature and personal observations of the
\end{abstract}


Actualización del conocimiento del género Cyttaria Berk. (Cyttariales, Ascomycota) en Chile. - Salazar-Vidal V.

author. A summary of the results of a recent study of the bioactive properties of the most consumed species in our country is also presented.

\section{INTRODUCCIÓN}

El género Cyttaria Berk. pertenece a la familia Cyttariaceae y es de carácter monotípico. Cuenta con 11 especies conocidas a nivel mundial, siete presentes en Sudamérica (Gamundí, 1971; 1991) y cuatro en Australasia (Rawlings, 1956), todas parásitas biótrofas exclusivas del género Nothofagus s.l., ocasionando tumores en sus hospederos de los cuales fructifican estromas (Figura 1).
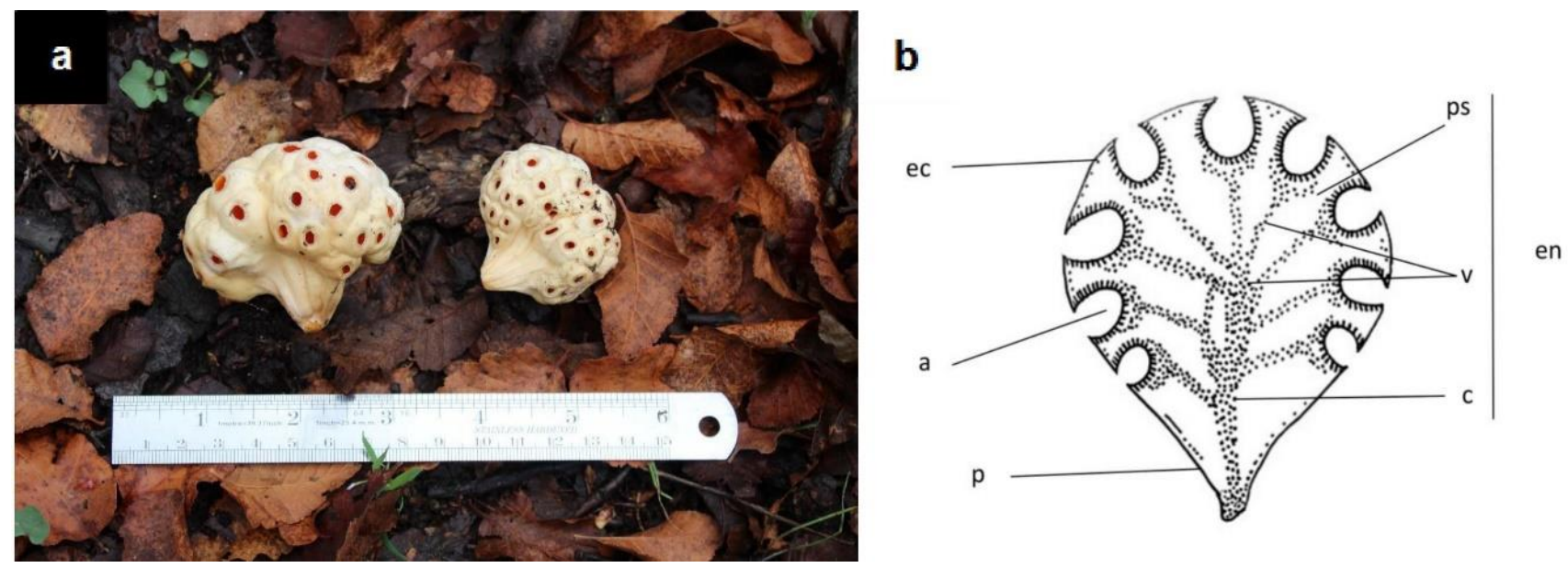

Figura 1. a) Estromas maduros de C. berteroi; b) Esquema general de las partes que conforman un estroma del género Cyttaria. ec: ectostroma, en: endostroma, a: apotecio, c: columela, ps: pseudotejido intersticial, v: venas, p: pie.

Existen numerosos trabajos taxonómicos sobre las especies sudamericanas del género Cyttaria (Barrera, 2004; Espinosa, 1926; Marchionatto, 1940; Gamundí, 1971, 1986; Gamundí et al., 2004; Sandoval-Leiva, 2012), en los cuales se da a conocer información relevante sobre estas especies y donde se evidencia la presencia en Chile de $C$. berteroi Berk., $C$. darwinii Berk., $C$. espinosae Lloyd, C. hariotii E. Fisch., $C$. hookeri Berk., C. johowii Espinosa y C. exigua Gamundí. La distribución del género Cyttaria está asociada a la presencia de Nothofagus, por este motivo en el sur de Sudamérica se encuentra distribuido en Chile y Argentina, pero también está presente en otros lugares del hemisferio sur como Australia y Nueva Zelanda.

En general, los estromas de estos hongos son carnosos a gelatinosos, esféricos a piriformes y en ellos se encuentran inmersos los apotecios, con ascos 8-esporados, cilíndricos, inoperculados, con presencia de un poro apical, paráfisis abundantes, pluriseptadas y filiformes, esporas uniseriadas, no septadas, hialinas, amarillentas cuando jóvenes, grises a negras cuando maduras, lisas a rugulosas y elípticas a subglobosas (Gamundí, 1971; 1986). A diferencia de la mayoría de los hongos, las paredes celulares de los estromas de C. hariotii y probablemente de otras especies del género, están conformadas por $\beta$-(1-3)-glucanos y carecen de quitina (Oliva et al., 1986).

Existe una asociación obligatoria entre especies del género Cyttaria y sus hospederos, que corresponden a árboles del género Nothofagus y a menudo se cita como un ejemplo clásico de cofilogenia en el que la biogeografía de un hongo es incluida (Peterson et al., 2010). En nuestro país, aún no se conocen todos los factores que influyen sobre los patrones de riqueza y composición de los macrohongos nativos, pero sí se sabe que la historia del género Nothofagus es clave en la comprensión 
Actualización del conocimiento del género Cyttaria Berk. (Cyttariales, Ascomycota) en Chile. - Salazar-Vidal V.

biogeográfica del Hemisferio Sur (Darlington, 1965; Steenis, 1971; Cracraft, 1975), con 35 especies vivientes, entre las cuales hay 10 representadas en Chile (Villagrán, 2018). Las asociaciones entre las especies de Cyttaria y Nothofagus (Figura 2), comúnmente no corresponden a una simple relación uno es a uno. Generalmente, una especie de Cyttaria se asocia con más de una (hasta 5) especies de
Nothofagus, que a su vez están asociados con más de una (hasta 4) especies de Cyttaria (Peterson et al., 2010). A diferencia de otras especies de Nothofagus presentes en nuestro país, el Ruíl (Nothofagus alesanndrii Espinosa) presenta una característica distintiva, es la única especie no parasitada por hongos del género Cyttaria (Santelices et al., 2018).

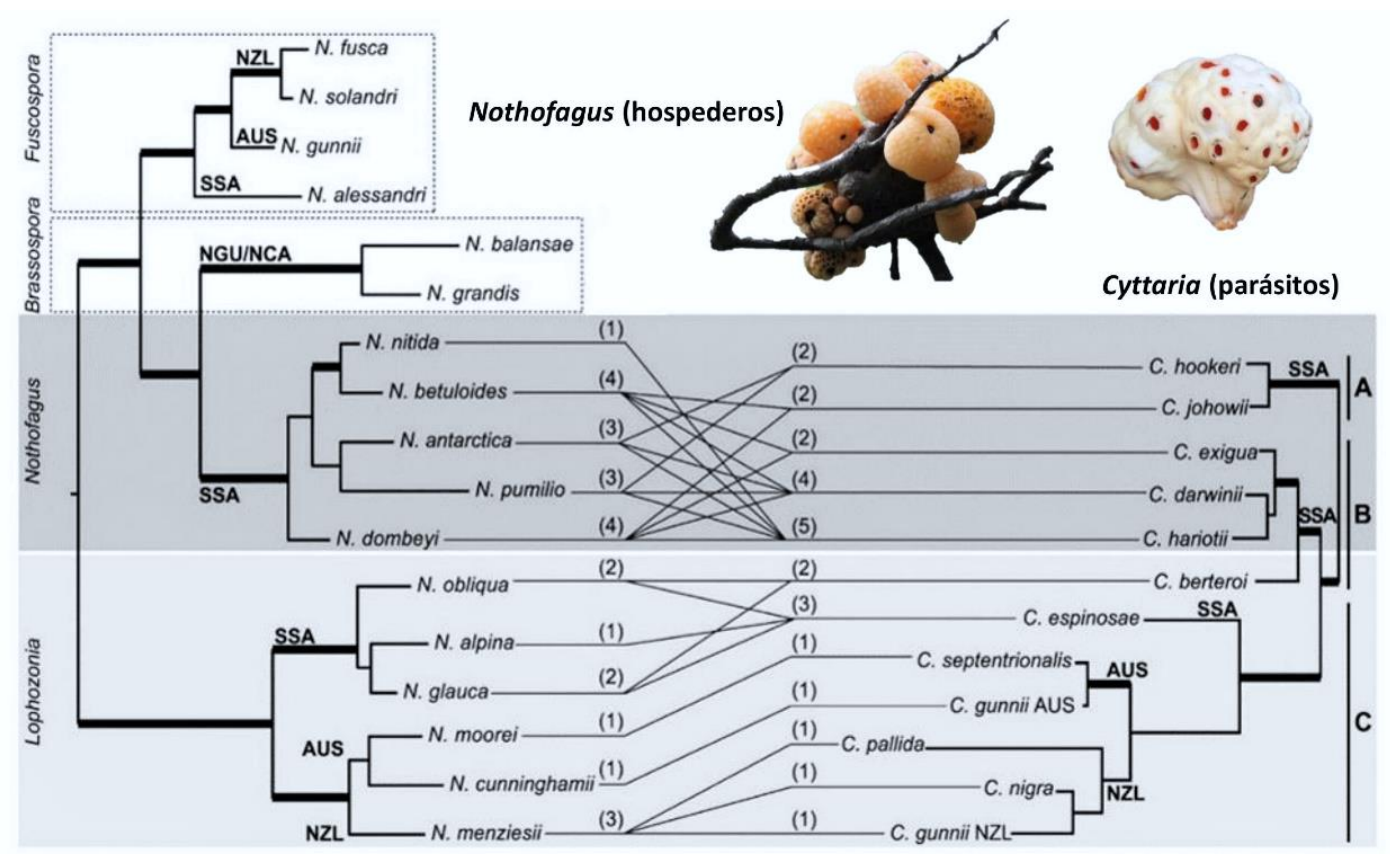

Figura 2. Relaciones entre los géneros Cyttaria y Nothofagus. Las líneas que conectan los árboles hospederos y sus hongos parásitos representan asociaciones; los números en paréntesis indican el número de asociaciones por taxón. Los recuadros sombreados con tonos grises indican los subgéneros de Nothofagus que albergan a Cyttaria spp. SSA: Sur de Sudamérica, AUS: Australia, NZL: Nueva Zelanda, NGU: Nueva Guinea, NCA: Nueva Caledonia (Adaptación de: Peterson et al., 2010).

\section{Descripción de las especies presentes en Chile}

Es importante reconocer las características distintivas de los hongos comestibles, esto se logra observando sus características macromorfológicas y microscópicas. Si bien todas las especies de Cyttaria presentes en nuestro país son consideradas como comestibles, a continuación, se describen macro y microscópicamente las especies presentes en Chile, considerando las descripciones realizadas por Gamundí (1971, 1986, 1991) y observaciones personales de la autora sobre su ecología, época de fructificación y comestibilidad.

Cyttaria berteroi Berk. Transactions of the Linnaean Society of London 19: 41 (1842).

Se denomina comúnmente como Pinatra o Curacucha (Figura 3a). Posee estromas maduros de gran tamaño, 2,5-12 $\mathrm{cm}$ de diámetro, gelatinosos, turbinados e irregularmente globosos, que emergen de tumores cilíndricos, con apotecios separados de boca pequeña, irregularmente circular o poligonal, con borde reflexo, delimitados superficialmente por 
Actualización del conocimiento del género Cyttaria Berk. (Cyttariales, Ascomycota) en Chile. - Salazar-Vidal V.

valéculas; sólidos o con cavidades irregulares y venas opacas, ramificadas irregularmente; sin espermogonios. De color amarillento a anaranjado en estado fresco y de color ferruginoso en seco. Presenta apotecios de 4-7 $\mathrm{mm}$ de profundidad, 7-12 $\mathrm{mm}$ de diámetro, urceolados, dispersos y escasos, con himenio anaranjado vivo en fresco, debido a un pigmento lipoide que contienen las paráfisis y los ascos; ascos 8-esporados, claviformes, inoperculados, adelgazándose hacia abajo para terminar en un pie bifurcado, con cilindro apical amiloide; ascosporas de 11-19 $\mu \mathrm{m}$ en diámetro, uniseriadas, cuando jóvenes subglobosas a elipsoides, al madurar más o menos cúbicas al interior del asco, irregularmente globosas a subpoliédricas al ser liberadas, quedando ocasionalmente adheridas unas con otras, con contenido granuloso y glóbulos amarillentos, episporio castaño, grueso y liso.

Se desarrolla sobre ramas de Nothofagus macrocarpa (A.DC.) F.M. Vázquez \& R.A. Rodr., Nothofagus glauca (Phil.) Krasser y Nothofagus obliqua (Mirb.) Oerst., fructificando durante la primavera, entre septiembre $y$ noviembre; con una distribución restringida desde la Región de Valparaíso hasta la Región de Los Lagos. Sus estromas maduros son grandes, con una consistencia carnosa, elástica y levemente acuosa. Tiene un olor y sabor levemente dulce. Suele ser muy codiciada y cada vez menos frecuente en el bosque, siendo una especie catalogada En Peligro (EN) de acuerdo al Reglamento de Clasificación de Especies según su Estado de Conservación (MMA), tanto para la Región de Valparaíso y Región Metropolitana, principalmente, por la tala de bosque nativo y recolección no sustentable.

Cyttaria espinosae Lloyd. Mycological Writings 5 (48): 673, t. 995, 998 (1917).

Recibe el nombre de Digüeñe común, Quireñe o Lihueñe (Figura 3b). Sus estromas maduros tienen un tamaño de 1,5-5 cm de diámetro, son carnosos, globosos y emergen de tumores esféricos, con muchos apotecios cercanos entre sí, de boca diagonal, separados por tabiques muy delgados; con base estéril blanca, hueco en la madurez y con endostroma de consistencia corchosa; con espermogonios abortados. De color crema-anaranjado a anaranjado en los 2/3 superiores cuando joven y de color anaranjadoocráceo cuando maduro. Presenta apotecios 4-6 $\mathrm{mm}$ de profundidad y de $3-5 \mathrm{~mm}$ de diámetro, prismáticos a piramidales, de aspecto aterciopelado y con himenio de color anaranjado; ascos 8-esporados, cilíndricos, paráfisis pluriseptadas, bifurcadas, ápice algo ensanchado, conteniendo muchas gútulas; paráfisis apicales robustas, cilíndricas, en empalizada laxa; ascosporas de 11-14 $\mu \mathrm{m}$ de diámetro, subglobosas, lisas, de paredes delgadas, conteniendo muchas gútulas, ocráceas a fumosas.

Crece sobre ramas de N. macrocarpa, N. glauca, N. obliqua, y Nothofagus alpina (Poepp. \& Endl.) Oerst. Fructifica durante la primavera, entre septiembre y noviembre; con una distribución restringida desde la Región de Valparaíso hasta la Región de Los Lagos. Sus estromas maduros, tienen una consistencia carnosa, blanda, algo seca y elástica. Olor y sabor agradable. Su recolección se ha mantenido a lo largo del tiempo, aumentando drásticamente en los últimos años. Esta especie de digüeñe es la más consumida a nivel nacional a lo largo de toda su distribución geográfica, siendo sus principales amenazas la sustitución de bosque nativo por plantaciones forestales y la sobreexplotación como Producto Forestal No Maderero (PFNM) con fines económicos.

Cyttaria hariotii E. Fisch. Bot. Ztg.: 816 (1888).

Se denomina comúnmente como Llao Llao, Llau Llau o Digüeñe del Coihue (Figura 3c). Presenta estromas maduros de $2-4,5 \mathrm{~cm}$ de diámetro, subglobosos con una base cónica, que emergen de tumores esféricos; con apotecios contiguos, separados por valéculas profundas, huecos en la columela, espermogonios superficiales, sobresalientes, de consistencia gelatinosa en estado fresco y córnea en seco. De color 
Actualización del conocimiento del género Cyttaria Berk. (Cyttariales, Ascomycota) en Chile. - Salazar-Vidal V.

amarillo-anaranjados y amarillo-ambarinos cuando maduros, con venación irregular. Presenta apotecios de 5-7 $\mathrm{mm}$ de profundidad, 3$8 \mathrm{~mm}$ de diámetro en la parte media y $2-5 \mathrm{~mm}$ de diámetro en la boca, caliciformes, con himenio anaranjado intenso; ascos 8-esporados, cilíndricos, achatados en el ápice, con un poro apical amiloide en estado inmaduro, con ápice desgarrado cuando dehiscente; paráfisis simples, pluriseptadas, catenuladas, conteniendo gránulos amarillentos; ascosporas de 12-15 $\mu \mathrm{m}$ de diámetro, uniseriadas, unicelulares, subglobosas, pero a veces poliédricas por la presión mutua que existe al interior del asco, con episporio grueso y áspero, ocre-oliváceo.

Se desarrolla sobre Nothofagus antarctica (G. Forst.) Oerst., Nothofagus pumilio (Poepp. \& Endl.) Krasser, Nothofagus dombeyi (Mirb.) Oerst., Nothofagus betuloides (Mirb.) Oerst. y Nothofagus nitida (Phil.) Krasser. Produce estromas durante primavera y verano, entre noviembre y enero; con una distribución desde la Región del Maule hasta la Patagonia. Sus estromas maduros tienen consistencia elástica y acuosa. Olor y sabor agradable, similar al durazno. Se consumen en poca cantidad, ya que poseen levaduras que provocan una fermentación espontánea, por lo que son utilizados para preparar chicha.

Cyttaria darwinii Berk. Transactions of the Linnaean Society of London 19: 37 (1842).

Recibe el nombre común de Pan de indio o Digüeñe del Ñirre (Figura 3d). Posee estromas de 2-4,5 cm de diámetro, globosos a globosoachatados, umbilicados en la base, que emergen de tumores esféricos; con apotecios ocupando $2 / 3$ a $3 / 4$ de su superficie, bastante separados entre sí por valéculas poco profundas; con espermogonios persistentes, de consistencia carnoso-gelatinosa en estado fresco, con el interior corchoso y el exterior córneo en seco. De color amarillo-anaranjado pálido en fresco y anaranjado-ferruginoso en seco; macizos. Presenta apotecios de 5-7 $\mathrm{mm}$ de profundidad y 3-5 $\mathrm{mm}$ de diámetro en la boca, caliciformes, con himenio de color anaranjado-amarillento a anaranjado vivo en fresco; ascos 8-esporados, cilíndricos, con un poro apical; paráfisis pluriseptadas, a veces constreñidas en los septos, bifurcadas o simples; ascosporas de 17-20 $\mu \mathrm{m}$ de diámetro, uniseriadas, unicelulares, subglobosas, muy apretadas en la parte superior del asco con una pared conspicua, cuando jóvenes conteniendo varias gútulas lipoides y entonces, apareciendo como cúbicas, de color fuliginoso claro.

Crece en ramas de $N$. pumilio, $N$. antarctica y $N$. betuloides. Sus estromas comienzan a aparecer durante la primavera (noviembre), con un periodo de maduración más largo que el resto de las especies de Cyttaria, permaneciendo en ramas sin hojas de ñirre y lenga durante invierno. Tiene una distribución que abarca desde la zona centro-sur hasta Tierra del Fuego. Sus estromas maduros tienen consistencia dura, con un olor y sabor suave.

Cyttaria johowii Espinosa. Bol Mus. Nac. Chile: 23 (1940).

Conocido comúnmente como Digüeñe mohoso del Coihue (Figura 3e). Presenta estromas de 1,5-2,5 cm de diámetro, moriformes, a napiformes, con 10-40 apotecios típicamente cupuliformes que ocupan 1/2 a 4/5 superiores del estroma, bastante separados por valéculas poco profundas, donde se alojan los espermogonios; columela castaña de donde irradian las venas, al ser hueco, contiene mucílago; consistencia gomosa en seco y resinosa al tacto. De color amarillo-anaranjado a ocre-ferruginoso en fresco, ocre-ferruginoso a tostado oscuro en seco. Presenta apotecios de 2-5 $\mathrm{mm}$ de profundidad y 1-4 mm de diámetro en la boca, caliciformes, en sección, aovados, con el himenio con margen delgado e inflexo, de color anaranjado en fresco; ascos 8-esporados, inoperculados, con un anillo apical; paráfisis pluriseptadas, simples o bifurcadas abajo, con contenido granuloso; ascosporas de 15-26 $\mu \mathrm{m}$ de diámetro, uniseriadas, globosas a subglobosas, 
Actualización del conocimiento del género Cyttaria Berk. (Cyttariales, Ascomycota) en Chile. - Salazar-Vidal V.

con contenido gutulado, episporio muy grueso, liso y asperulado de color fuliginoso.

Se desarrolla sobre ramas de $N$. dombeyi y $N$. betuloides. Sus estromas aparecen en el otoño (marzo-abril) madurando en la primavera tardía y verano (noviembre-enero). Al poco tiempo de madurar caen al suelo. No forma tumores notorios, más bien una ligera hinchazón. Se distribuye desde Talca hasta Tierra del Fuego. Tiene un olor y sabor suave, en este último caso, levemente dulce. Se puede decir que es la especie de Cyttaria más difícil de observar a simple vista en nuestro país.

Cyttaria hookeri Berk. Transactions of the Linnean Society 20: 452, t. 162:1 (1847).

Recibe el nombre común de Digüeñe mohoso del Nirre (Figura 3f). Tiene estromas de $1-2 \mathrm{~cm}$ de diámetro, turbinados, con un umbón apical donde se agrupan los espermogonios persistentes, crecen a partir de tumores cilíndricos; con apotecios en número de 1-8 ocupando la parte superior; macizos e interiormente de color amarillento pálido, consistencia gomosa, elástica y firme. De color ocre ferruginoso en fresco, en seco castañorojizo, muy duro y resinoso al tacto. Presenta apotecios de 4,5 $\mathrm{mm}$ de profundidad y 3-4,5 $\mathrm{mm}$ de diámetro, en la parte media y 1,5-3 $\mathrm{mm}$ de diámetro en la boca, caliciformes, con margen inflexo, delgado y estriado transversalmente, himenio castaño-oliváceo en fresco y castañooscuro en seco; ascos 8-esporados, cilíndricos, inoperculados; paráfisis simples, pluriseptadas, moniliformes en el ápice, abajo catenuladas y contenido granuloso amarillento, con paredes con gránulos con pigmento castaño; ascosporas de 17-19 $\mu \mathrm{m}$ diámetro, uniseriadas, , apisporio liso a asperulado, grueso, subglobosas a ovoides, a veces con un apículo, con muchas gútulas; de color castaño pálido.

Crece sobre ramas juveniles de $N$. antarctica y $N$. pumilio. Sus estromas comienzan a formarse en enero y maduran en la primavera del mismo año (septiembre-octubre). Se encuentra distribuida desde la Región de Ñuble hacia el sur. Tiene una consistencia dura y un sabor levemente amargo.

Cyttaria exigua Gamundí. Darwiniana 16: 495 (1971).

Nombre común desconocido (Figura 3g). Posee estromas de tamaño pequeño $0,7-2 \mathrm{~cm}$ de diámetro, globoso-achatados, umbilicados en la base, distribuidos en grupos; espermogonios presentes o ausentes. De color amarillento pálido con tinte rosado en estado juvenil y violáceo claro cuando maduros. Con apotecios en número de 12-20 muy separados entre sí y de bocas circulares hundidos en el estroma, ocupando entre $3 / 4$ a $4 / 5$ de la parte superior. Presenta apotecios de 1,6-3 mm de profundidad y 1,8-4 $\mathrm{mm}$ de diámetro, intensamente caliciformes, con margen elevado e inflexo, de consistencia carnoso-gelatinosa, elástica y firme, himenio amarillo-anaranjado en fresco y anaranjado intenso en seco; ascos 8-esporados, cilíndricos, inoperculados, con un cilindro apical; paráfisis filiformes, pluriseptadas, hialinas, bifurcadas, delgadas; ascosporas de 14-17 $\mu \mathrm{m}$ de diámetro, uniseriadas, globosas o deformadas en los polos por la presión entre los polos apical y distal, claro-oliváceas, lisas, conteniendo varias gútulas.

Se desarrolla en hileras longitudinales sobre ramas delgadas de $N$. dombeyi y $N$. betuloides. Sus estromas aparecen en época estival y tienen un corto periodo de desarrollo, que comienza en primavera y termina a mediados de verano (diciembre-enero). Se distribuye entre la Región de Los Lagos y Región de Magallanes. Actualmente, se encuentra catalogada como Preocupación menor (LC), principalmente, por contar con escasos registros. No se conocen antecedentes sobre su comestibilidad, pero dado su pequeño tamaño, no se considera relevante en este aspecto. 
Actualización del conocimiento del género Cyttaria Berk. (Cyttariales, Ascomycota) en Chile. - Salazar-Vidal V.
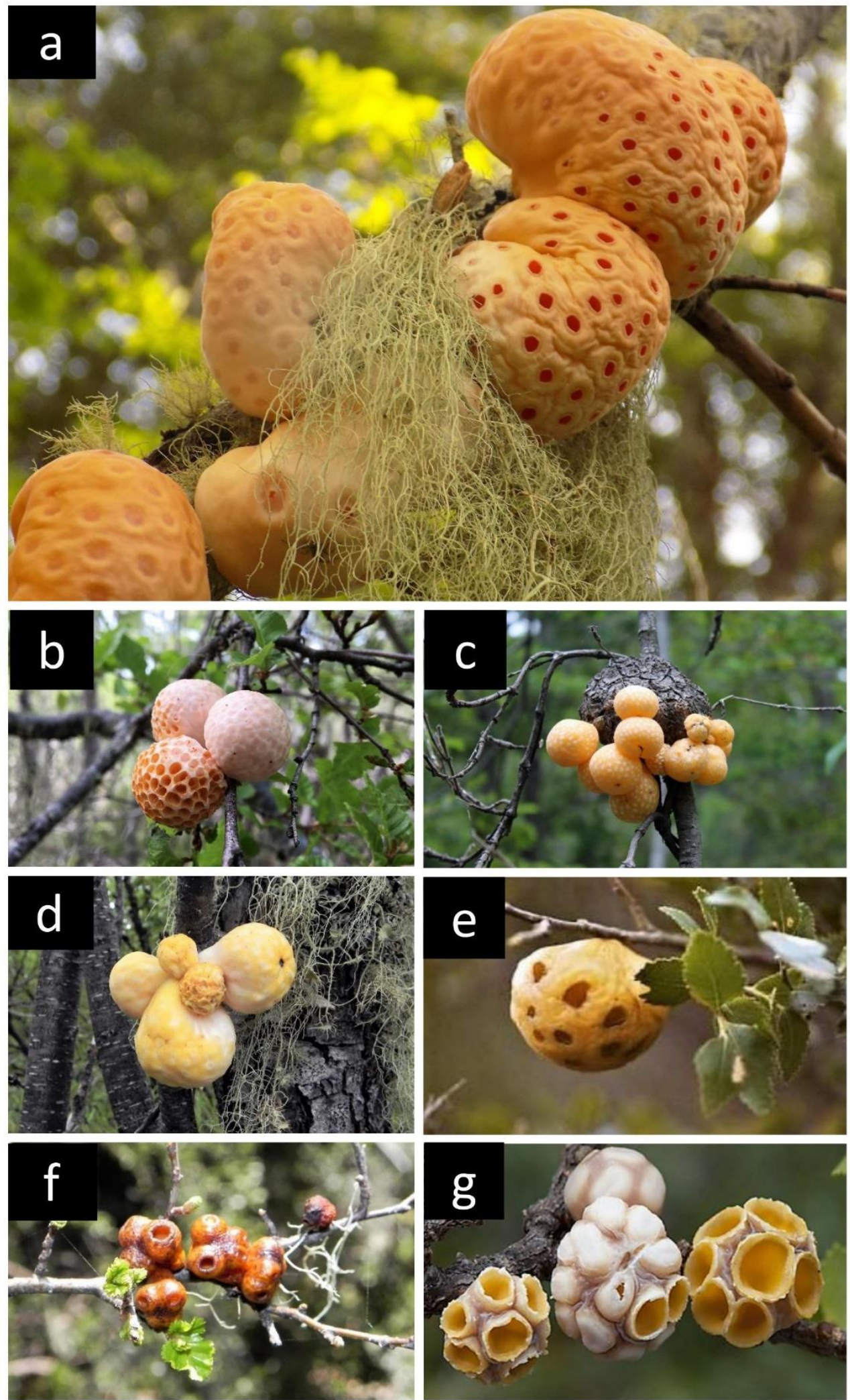

Figura 3. Especies del género Cyttaria presentes en Chile. a) C. berteroi, b) C. espinosae, c) C. hariotii, d) C. darwinii, e) C. johowii, f) C. hookeri, g) C. exigua. 
Actualización del conocimiento del género Cyttaria Berk. (Cyttariales, Ascomycota) en Chile. - Salazar-Vidal V.

\section{Usos tradicionales y propiedades bioactivas de las especies más consumidas}

Los Productos Forestales No Madereros (PFNM) en nuestro país son definidos como "aquellos bienes de origen biológico distinto de la madera, procedentes de los bosques, de otros terrenos arbolados y de árboles situados fuera de los bosques, independiente de su naturaleza" (INFOR, 2009). En los últimos años, los PFNM han adquirido un interés considerable en todo el mundo, ya que se está reconociendo su importancia para contribuir a la conservación de la diversidad biológica y, además, se ha comprobado que su recolección y consumo mejora la calidad de vida de las personas.

La recolección de PFNM es una actividad ancestral donde los hongos silvestres comestibles (HSC) han tenido gran importancia. Según López \& Fuenzalida (1998) numerosos hongos son recolectados por los mapuches como alimento, dentro de los que destacan especies del género Cyttaria, conocidas vulgarmente como digüeñes, quireñes, entre otros nombres comunes, que se distribuyen en Chile desde la zona central hasta la Patagonia. La práctica de su recolección como alimento se remonta a los primeros habitantes de estas tierras y aún son recolectados. Los cuerpos fructíferos de estos hongos en un comienzo eran considerados "frutos" del árbol hospedero y no solamente se consumían en estado fresco, sino también se hacían fermentar para obtener un brebaje alcohólico conocido como "chicha" (Mösbach, 1991).

Las especies del género Cyttaria han sido recolectadas desde la prehistoria por varias tribus amerindias y usados como alimento (Domínguez, 2010). El pueblo Mapuche conformado originalmente por cazadores, recolectores y agricultores, vivía en un territorio coincidente con los bosques de Nothofagus del centro y sur de Chile, en los cuales recolectaba digüeñes. En la Región de Los Ríos y Región de Los Lagos, la recolección de PFNM, en especial, de HSC es una tradición que se remonta a mucho tiempo atrás. De hecho, para los Huilliches los hongos comestibles formaban parte importante de su dieta (Sepúlveda, 2005; Egert \& Godoy, 2017).

Otros autores se refieren al consumo de los digüeñes por etnias que habitaban Tierra del Fuego, por ejemplo: $C$. darwinii fue consumido por los Selknam (Onas) y también era recolectada por los Kawashkar (Alacalufes) (Emperaire, 1963) y Yámanas (Chapman, 1987). Mientras que $C$. hookeri, conocido como "Assuim" o "Uaíaca" en esta zona geográfica, también era recolectado, con un sabor ligeramente amargo e inoloro en estado fresco, siendo consumido preferentemente deshidratado (Muñoz et al., 1981).

Muñoz et al., (1981) incluyen a $C$. berteroi, $C$. darwinii, C. espinosae, C. hariotii y $C$. hookeri entre las especies comestibles de este género, aunque hoy en día gracias a distintas investigaciones, se sabe que todas las especies de Cyttaria presentes en Chile son comestibles, algunas en menor cantidad que otras por su consistencia y sabor. Su recolección para el consumo y venta directa, continúa siendo una actividad conveniente para la población rural de la zona centro-sur de Chile, donde los digüeñes son vendidos en mercados y ferias locales. Todas las Cyttaria spp. comercializadas son recolectadas en los bosques dominados por Nothofagus. La especie más común es $C$. espinosae, que habitualmente se encuentra en renovales de $N$. macrocarpa y $N$. obliqua, mientras que $C$. berteroi es común en árboles maduros de $N$. macrocarpa, $N$. glauca y $N$. obliqua.

Aunque cada vez son más las personas que consumen digüeñes en Chile, todavía se conoce poco acerca de su valor nutritivo y compuestos bioactivos que presenten actividad biológica y/o farmacológica relevante, destacando los estudios realizados por los autores Ipinza et al., (1989) y Schmeda-Hirschmann et al., $(1999,2001)$ en la Región Metropolitana y Región del Maule, respectivamente. Por el contrario, en Argentina se han realizado varios trabajos examinando ejemplares del género Cyttaria, los que han llevado a aislar y determinar la estructura de 
Actualización del conocimiento del género Cyttaria Berk. (Cyttariales, Ascomycota) en Chile. - Salazar-Vidal V.

compuestos solubles en agua o solventes polares, formados por un número elevado de moléculas de azúcares simples. La composición de los polisacáridos de $C$. hariotii y $C$. johowii fue investigada por Femández-Cirelli \& Lederkremer (1974), Waksman et al., (1975) y Waksman et al., (1977). Mientras que Femández-Cirelli et al., (1989), analizaron el efecto de los cambios estructurales sobre la acción antitumoral de los glucanos de $C$. hariotii y C. darwinii.

Diversos estudios han permitido determinar que los hongos comestibles pueden tener diferentes acciones terapéuticas, tales como un efecto: antitumoral, inmunomodulador, antidiabético, hipocolesterolémico, hepatoprotector, antiviral, antibacteriano y antiparasitario (Albertó, 2008; Díaz, 2016). Los hongos medicinales se caracterizan por tener materiales en la pared celular y metabolitos secundarios que tienen un rango más amplio de propiedades farmacológicamente activas en comparación con hongos que sólo son comestibles (Lindequist et al., 2005; Rivera et al., 2017).

Entre las sustancias biológicamente activas presentes en los hongos, los compuestos fenólicos han atraído la atención debido a sus excelentes propiedades antioxidantes, antiinflamatorias o antitumorales (Puttaraju et al., 2006; Cano-Estrada \& Romero-Bautista, 2016). El estudio de la actividad antioxidante se considera importante, ya que permite combatir los radicales libres que provocan gran parte de las enfermedades degenerativas, ocasionando el deterioro de la calidad de vida de las personas o la muerte (Badarinath et al., 2010; Bahadori et al., 2019). Los compuestos fenólicos poseen una fuerte actividad antioxidante, lo que ha sido demostrado en numerosos trabajos, principalmente in vitro, desde extractos acuosos y metanólicos de hongos comestibles (Mau et al., 2001, Heleno et al., 2012).

De acuerdo a Schmeda-Hirschmann et al., (1999), a lo largo de la historia miles de especies de plantas y hongos han sido utilizados para la alimentación y con fines medicinales, lo que no sólo implica un aporte nutricional, sino también el ingreso al organismo de compuestos que influyen positivamente en nuestra salud. Como se mencionó anteriormente, la mayor parte de las investigaciones relacionadas con el género Cyttaria en Chile y otros países, está referida a su taxonomía, evolución y ecología (Espinosa, 1926; Marchionatto, 1940; Rawlings, 1956; Gamundí, 1971; Ipinza et al., 1989; Peterson et al., 2010). Existen estudios previos sobre la composición químico-nutricional de Cyttaria spp. realizados por Schmeda-Hirschmann et al., (1999, 2001) con especies recolectadas mayormente en la Región del Maule, zona central de Chile, donde se investigó la presencia de diversos compuestos con actividad antimicrobiana, antiproliferativa y antitumoral.

Recientemente, Salazar-Vidal (2019) realizó un estudio junto al equipo del Laboratorio de Química de Productos Naturales ubicado en la Facultad de Cs. Naturales y Oceanográficas de la Universidad de Concepción, donde se logró determinar la composición nutricional que aportan las especies de Cyttaria más consumidas en Chile en base a \% (g/100g) en peso seco mediante un análisis proximal. Los resultados evidenciaron diferencias entre $C$. espinosae y $C$. hariotii, donde se pudo observar que el contenido proteico fue mayor para $C$. espinosae con un valor promedio de $20 \%$ en peso seco, llegando a ser casi cinco veces mayor que en la especie $C$. hariotii que presentó un valor promedio de proteínas igual a un $4,1 \%$ en peso seco, coincidiendo con lo reportado por Schmeda-Hirshmann et al., (1999), que obtuvieron valores similares en cuanto al contenido de proteínas presentes en las especies recolectadas en la Región del Maule, con un valor promedio de $18,5 \%$ en peso seco para $C$. espinosae y de $7,5 \%$ en peso seco para $C$. hariotii, respectivamente. Asimismo, Toledo et al., (2016) reportaron 3,3\% de proteínas al analizar muestras de $C$. hariotii en la Patagonia, mientras que Inzunza (2016) obtuvo un porcentaje de proteínas igual a un $20 \%$ para $C$. espinosae y un $13 \%$ para $C$. hariotii, aproximadamente, analizando muestras provenientes de la Región del Ñuble. Las 
Actualización del conocimiento del género Cyttaria Berk. (Cyttariales, Ascomycota) en Chile. - Salazar-Vidal V.

proteínas y carbohidratos son los dos componentes principales presentes en los hongos comestibles (Kalac `2, 2009), siendo estos dos parámetros donde se observó mayores diferencias entre las especies estudiadas: $C$. espinosae y C. hariotii.

Salazar-Vidal (2019) junto a colaboradores, también realizó estudios con extractos metanólicos entre dos especies de Cyttaria, donde se obtuvo una mayor actividad antioxidante para los extractos de $C$. hariotii en comparación con los extractos de C. espinosae, representando una fuente natural de compuestos funcionales que ayudan a prevenir o retrasar el daño que producen los radicales libres a las células del cuerpo. Además, en esta investigación, los polisacáridos totales extraídos de $C$. hariotii y $C$. berteroi presentaron diferencias en la actividad citotóxica en líneas tumorales de leucemia humana, cáncer colon y cáncer de mama, donde los polisacáridos de $C$. hariotii presentaron una alta citotoxicidad en la línea celular de leucemia humana. SchemedaHirshmann et al., (2001), evaluó el efecto inmunomodulador de extractos hidrosolubles de cuatro especies de Cyttaria: $C$. berteroi, $C$. darwinii, $C$. espinosae y $C$. hariotii en ratones con linfoma L5178Y, administrando oralmente de $100 \mathrm{mg}$ de extracto durante 7 días, donde en el modelo de hipersensibilidad de tipo retardado, sólo $C$. hariotii pudo modificar la respuesta inmune, sugiriendo que la ingesta de esta especie puede mejorar el sistema inmunológico de los consumidores.

\section{OBSERVACIONES}

Hasta la fecha, en nuestro país han sido registradas siete especies del género Cyttaria, las cuales también están descritas para Argentina. De acuerdo a la literatura disponible, las especies más consumidas en Chile son: $C$. berteroi, $C$. espinosae y $C$. hariotii. Este género de hongos parásitos no sólo es importante desde el punto de vista taxonómico, ecológico y etnomicológico, sino también como una fuente de compuestos bioactivos, que si son mejor estudiados podrían llevar al aprovechamiento del potencial medicinal de este PFNM, promoviendo su recolección sustentable y contribuyendo a su conservación.

De acuerdo al Reglamento de Clasificación de Especies según su Estado de Conservación del Ministerio del Medio Ambiente, C. berteroi se encuentra catalogada En Peligro (EN) para la Región de Valparaíso y Región Metropolitana, siendo sus principales amenazas actuales la restricción de su distribución geográfica, la tala del bosque nativo dominado por Nothofagus spp. y la sobreexplotación como PFNM, debido al aumento de su recolección para venta y consumo, lo que podría extrapolarse a otras especies del género, especialmente, a $C$. espinosae por ser una de las especies más consumidas a lo largo de su distribución. Otra especie del género Cyttaria que está catalogada actualmente en una categoría de conservación en nuestro país es C. exigua, pero como Preocupación menor (LC), por contar con escasos registros.

Muchas especies de hongos aún no se han estudiado en Chile, debido a las condiciones de aislamiento biogeográfico que favorecen la presencia de especies endémicas, por lo es sumamente necesario realizar más investigaciones para descubrir los beneficios que estos organismos pueden tener para nuestra salud, los que no sólo deben basarse en compuestos que tengan un buen aporte nutricional para nuestra dieta, sino también en elementos y productos naturales que permitan, ya sea prevenir o tratar enfermedades degenerativas.

\section{AGRADECIMIENTOS}

Agradezco al Prof. Waldo Lazo y a la Dra. Andrea Romero del Instituto de Micología y Botánica de la Universidad de Buenos Aires por facilitarme bibliografía relevante del género Cyttaria. A la Dra. Irma Gamundí por su enorme contribución al conocimiento del Orden Cyttariales a través de sus publicaciones. A Eitel Thielemann y Pablo Sandoval por contribuir con 
Actualización del conocimiento del género Cyttaria Berk. (Cyttariales, Ascomycota) en Chile. - Salazar-Vidal V.

fotografías. Al equipo del Laboratorio de Química de Productos Naturales de la Universidad de Concepción por su apoyo en los experimentos que he realizado estos últimos años sobre la composición químico-nutricional y propiedades bioactivas de las especies más consumidas del género Cyttaria. Al Dr. Mario Aranda del Departamento de Farmacia de la Pontificia Universidad Católica de Chile.

\section{REFERENCIAS}

Albertó, E. 2008. Cultivo intensivo de los hongos comestibles: Cómo cultivar Champiñoñes, Gírgolas, Shiitake y otras especies. Editorial Hemisferio Sur S.A. 265 pp.

Badarinath, A., Rao, M., Chetty, C., Ramkanth, S., Rajan, T. \& K. Gnanaprakash, 2010. A review on in-vitro antioxidant methods: comparisons, correlations and considerations. International Journal of Pharm Tech Research, 2: 1276-1285.

Bahadori, M., Sarikurkcu, C., Yalcin, O., Cengiz, M. \& H. Gungor. 2019. Metal concentration, phenolics profiling, and antioxidant activity of two wild edible Melanoleuca mushrooms ( $M$. cognata and $M$. stridula). Microchemical Journal, 150, 104172.

Barrera, E. 2004. Especies chilenas Cyttaria Berkeley (Cyttariaceae). Chagual 2: 62-65.

Cano-Estrada, A. \& L. Romero-Bautista. 2016. Valor económico, nutricional y medicinal de hongos comestibles silvestres. Revista Chilena de Nutrición, 43(1): 75-80.

Chapman, A. 1987. La Isla de los Estados en la Prehistoria. Editorial Universitaria de Buenos Aires (Eudeba), Buenos Aires, Primeros datos arqueológicos. Colección Temas.

Cracraft, J. 1975. Historical biogeography and Earth history-perspectives for a future synthesis.
Annals of the Missouri Botanical Garden, 62: 227-250.

Darlington, P. 1965. Biogeography of the southern end of the world; distribution and history of far-southern life and land, with an assessment of continental drift. Cambridge, Massachusetts: Harvard Univ. Press. 236 pp.

Díaz, M. 2016. Alimentos funcionales obtenidos a partir de hongos nutraceúticos. Facultad de Tecnología, Química Industrial. Universidad Tecnológica de Pereira.

Domínguez, E. 2010. Flora de interés etnobotánico usada por los pueblos originarios: Aónikenk, Selk'nam, Kawésqar, Yagan y Haush en la Patagonia Austral. Dominguezia, 26(2): 19-29.

Egert, M., \& M. Godoy. 2017. Semillas, cultivos y recolección al interior de una familia mapuche huilliche en Lumaco, Lanco, Región de los Ríos, Chile. Revista Austral de Ciencias Sociales, (14): 51-70.

Emperaire, J. 1963. Los Nómades del Mar. Edit. Universidad de Chile, Santiago de Chile.

Espinosa, M. 1926. Los hongos del género Cyttaria. Revista Chilena de Historia Natural, 30: 206-256.

Fernández-Cirelli, A. \& R. de Lederkremer. 1974. Structure of a polysaccharide from Cyttaria harioti Fischer. Acetolysis studies. Anales de la Asociación Química Argentina, 62: 141-145.

Fernández-Cirelli, A., Oliva, E. \& R. de Lederkremer. 1989. Occurrence of Darabinohexulosonic acid in polysaccharides of Cyttaria species. Phytochemistry, 28: 16451647. 
Actualización del conocimiento del género Cyttaria Berk. (Cyttariales, Ascomycota) en Chile. - Salazar-Vidal V.

Gamundí, I. 1971. Las Cyttariales sudamericanas (Fungi-Ascomycetes).

Darwiniana, 16(3-4): 461-510.

Gamundí, I. 1986. Fungi, Ascomycetes. Cyttariales, Helotiales: Geoglossaceae, Dermateaceae. Flora criptogámica de Tierra del Fuego. 10(4): 1-126.

Gamundí, I. 1991. Review of recent advances in the knowledge of the Cyttariales. Systema Ascomycetum, 10: 69-77.

Gamundí, I., Minter, D., Romero, A., Barrera, V., Giaiotti, A., Messutti, M. \& M. Stecconi. 2004. Checklist of the Discomycetes (Fungi) of Patagonia, Tierra del Fuego and adjacent Antarctic areas. Darwiniana, 42(1-4): 63-164.

Heleno, S. A., Barros, L., Martins, A., Queiroz, M. J. R., Santos-Buelga, C., \& Ferreira, I. C. 2012. Phenolic, polysaccharidic, and lipidic fractions of mushrooms from Northeastern Portugal: chemical compounds with antioxidant properties. Journal of Agricultural and Food Chemistry, 60(18): 46344640 .

INFOR. 2009. Exportaciones forestales de productos no madereros. Boletín $\mathrm{N}^{\circ} 4$, septiembre 2009. 18 pp.

Inzunza, K. 2016. Propiedades bioactivas de dos especies de Cyttaria (digüeñes) ( $C$. espinosae y $C$. hariotii) y su caracterización nutricional. Facultad de Ingeniería Agrícola, Universidad de Concepción.

Ipinza, R., Pérez, F. \& A. Kappes. 1989. Cyttaria espinosae Lloyd., un hongo de interés en fitopatología, alimentación y evolución de los bosques de Nothofagus en Chile. Boletín Sociedad Micológica de Madrid, 13: 31-47.

Kalač, P. 2009. Chemical composition and nutritional value of European species of wild growing mushrooms: A review. Food chemistry, 113(1): 9-16.

Lindequist, U., Niedermeyer, T. \& W. Julich. 2005. The pharmacological potential of mushrooms. Evidence-Based Complementary and Alternative Medicine 2: 285-99.

López, L. \& F. Fuenzalida. 1998. Algunos problemas identificados en la comercialización de productos provenientes del bosque nativo. Proyecto Manejo Sustentable del Bosque Nativo. CONAF.

Marchionatto, J. 1940. Las especies de Cyttaria y Cytariella en la Argentina Darwiniana, 4(1): 9-32.

Mau, JL., Lin, HC., Ma, JT. \& SF. Song. 2001. Non-volatile taste components of several specialty mushrooms. Food Chemistry, 73: 4616.

Möshach, E. 1991. Botánica Indígena de Chile. C. Aldunate \& C. Villagrán, ed., Museo Chileno de Arte Precolombino, Fundación Andes y Editorial Andrés Bello, Santiago de Chile.

Muñoz, M., Barrera, E. \& I. Meza. 1981. El uso medicinal y alimenticio de plantas nativas y naturalizadas en Chile. Publicación ocasional $\mathrm{N}^{\circ}$ 33, Museo Nacional de Historia Natural, Santiago de Chile.

Oliva, E., Fernandez-Cirelli, A. \& R. Lederkremer. 1986. Chemical composition of the cell Wall of the tree fungus Cyttaria hariotii Fischer. Experimental Mycology, 10: 150-156.

Peterson, K., Pfister, D. \& C. Bell. 2010. Cophylogeny and biogeography of the fungal parasite Cyttaria and its host Nothofagus, Southern Beech. Mycologia, 102(6): 1417-1425.

Puttaraju, N., Venkateshaiah, S., Dharmesh, S., Urs, S. \& R. Somasundaram. 2006. Antioxidant activity of indigenous edible 
Actualización del conocimiento del género Cyttaria Berk. (Cyttariales, Ascomycota) en Chile. - Salazar-Vidal V.

mushrooms. Journal of Agricultural and Food Chemistry, 54: 9764-9772.

Rawlings, G. $1956 . \quad$ Australasian Cyttariaceae.Transactions and Proceedings of the Royal Society of New Zealand, 8(1): 19-28.

Rivera, O., Albarracín, W. \& M. Lares. 2017. Componentes Bioactivos del Shiitake (Lentinula edodes Berk. Pegler) y su impacto en la salud. Archivos Venezolanos de Farmacología y Terapéutica, 36(3): 67-71.

Salazar-Vidal, V. 2019. Comparación de parámetros químico-nutricionales de las especies del género Cyttaria más consumidas en Chile. Facultad de Ciencias Forestales, Universidad de Concepción.

Sandoval-Leiva, P. 2012. Acerca de Cyttaria exigua Gamundí en Chile. Boletín Micológico, 27(2): 61-64.

Santelices, R., Espinoza, S., Cabrera, A. \& C. Magni. 2018. Gestión de riesgo como herramienta para la conservación de los bosques de Nothofagus alessandrii, una especie en peligro de extinción de Chile central. Interciencia, 43(2): 144-150.

Schmeda-Hirschmann, G., Razmilic, I., Reyes, S., Gutiérrez, M. \& J. Loyola. 1999. Biological Activity and Food Analysis of Cyttaria spp. (Discomycetes). Economic Botany, Vol. 53 (1): 30-40.

Schmeda-Hirschmann, G., VillaseñorGarcía, M., Lozoya, X. \& A. Puebla-Pérez. 2001. Immunomodulatory activity of Chilean Cyttaria species in mice with L5178Y lymphoma. Journal of Ethnopharmacology, 77(2-3): 253-257.

Sepúlveda, J. 2005. Principios de alimentación mapuche como un aporte a la soberanía alimentaria. Centro de educación y tecnología para el desarrollo del sur (CETSUR), Temuco.
Steenis, C. 1971. Nothofagus, key genus of plant geography, in time and space, living and fossil, ecology and phylogeny. Blumea, 19: 65-98.

Toledo, C., Barroetaveña, C., Fernandes, A., Barros, L. \& I. Ferreira. 2016. Chemical and antioxidant properties of wild edible mushrooms from native Nothofagus spp. forest, Argentina. Molecules, 21(9), 1201.

Villagrán, C. 2018. Biogeografía de los bosques subtropical-templados del sur de sudamérica. Hipótesis históricas. Magallania (Punta Arenas), 46(1): 27-48.

Waksman, N., Svec, B., Cirelli, A. \& R. de Lederkremer. 1975. Identification and quantitative determination of D-arabinohexulosonic acid in Cyttaria species. Phytochemistry, 14: 1009-1010.

Waksman, N., de Lederkremer, R. \& A. Cerezo. 1977. The structure of an Alpha-Dglucan from Cyttaria harioti Fischer (Fungi). Carbohydrate Research, 59: 505-515. 